\title{
Environmental Information Systems on the Internet: A Need for Change
}

\author{
Sven Schade $^{1}$, Barbara Fogarty ${ }^{2}$, Michael Kobernus ${ }^{3}$, Katharina Schleidt ${ }^{4}$, \\ Paul Gaughan ${ }^{5}$, Paolo Mazzetti ${ }^{6}$, and Arne-Jørgen Berre ${ }^{7}$ \\ ${ }^{1}$ Institute for Environment and Sustainability, \\ Joint Research Centre of the European Commission, Ispra, Italy \\ sven.schade@jrc.ec. europa.eu \\ ${ }^{2}$ Dublin City University \\ barbara. fogarty@dcu. ie \\ ${ }^{3}$ Norsk Institutt for Luftforskning, Norway \\ mike.kobernus@nilu.no \\ ${ }^{4}$ Umweltbundesamt Östereich, Austria \\ katharina.schleidt@umwel tbundesamt.at \\ ${ }^{5}$ Marine Institute, Ireland \\ paul.gaughan@marine.ie \\ ${ }^{6}$ Consiglio Nazionale delle Ricerche, Italy \\ mazzetti@imaa.cnr.it \\ ${ }^{7}$ Stiftelsen SINTEF, Norway \\ arne.j.berreasintef.no
}

\begin{abstract}
The cost effective delivery of scientific and policy requirements is a key driver for the realization of global sustainability research, integrated assessment and supporting innovative systems. The next generation of geospatial information infrastructures is proposed as a possible solution. Still, questions such as 'what does all this mean to environmental information systems' and 'what is expected to change', have only partially been answered. In this paper, we describe the recent challenges for eEnvironment services in Europe, specify desired capabilities and derive according requirements. We identify affected stakeholder communities and depict their involvement in the overall value chain of environmental knowledge generation. Specific examples illustrate individual needs, while a derived description of the value chain indicates more general outcomes. Developmental requirements of future information systems are discussed. The presented work answers the questions above by bridging the gab between stakeholder needs, Information and Communication Technology (ICT) development and higher level concepts, such as Digital Earth and Future Internet.
\end{abstract}

\section{Introduction}

The International Council for Science (ICSU) identified five scientific priorities, or Grand Challenges, in global sustainability research, including (i) development of observation systems needed to manage global and regional environmental change; (ii) improvement of the usefulness of forecasts of future environmental conditions and 
their consequences for people; and (iii) investigation of institutional, economic and behavioral responses that can enable effective steps toward global sustainability [1]. A next generation of geospatial information infrastructures (Digital Earth) has been proposed as a possible solution, which provides more dynamic systems, new sources of information, and stronger capacities for their integration [2].

With the Europe 2020 strategy [3], especially under the umbrella of the Digital Agenda [4] and Innovation Union [5] flagship initiatives, the EU provided the required policy context for addressing the above mentioned Grand Challenges and for implementing a Digital Earth. The advent of Digital Science 2030 [6] indicates a growing importance. Here, among others, ICT-intense domains are challenged to develop innovative applications based on technological enablers, such as manifold sensors, simulation tools and scientific data infrastructures. The evolution of the Internet (Future Internet) [7] shall play a major role.

Yet, many questions remain unanswered. How do these high level concepts reflect reality? What does all this mean to environmental information systems? What is expected to change? Before these topics can be properly addressed, we have to carefully describe the recent challenges for eEnvironment services in Europe [8], specify desired capabilities and derive according requirements. Only if these are met, can we identify affected stakeholder communities, discuss suitable implementation frameworks and suggest sustainable development strategies.

This paper provides such groundwork. We review cases from three environmental spheres (terrain, atmosphere and marine) to illustrate the richness of applications across the borders of EU Member States, and the variety of stakeholders involved; examples range from classical monitoring and reporting, via downscaling of global environmental models to individual needs, to the inclusion of user contributed content into the value chain of environmental knowledge generation. We furthermore introduce some 'science fiction' services, in which we think outside the box of expected environmental applications.

The remainder of this paper is structured as follows. The next section presents viewpoints from three environmental spheres. It also includes a set of (science fiction) applications, which might become reality in the advent of new technologies and user communities. Thereafter, section 3 discuses possible generalization of the requested eEnvironment services and derives a common description of the underlying value chain. A suitable framework for developing required information systems is depicted in section 4. Given future developments in Information and Communication Technology (ICT), we put emphasis on the future of the Internet (or Future Internet). The paper concludes with a summary of our findings and an outlook to required future activities.

\section{2 eEnvironment Services for Three Spheres}

This section presents three concrete cases in which environmental information systems have to change in order to meet arising needs. Each section follows a similar structure. The overall context is defined first; followed by a discussion on the intended goals, steps to achieve them, derived case-specific requirements, and the resulting stakeholders. We specifically include issues of scalability, either in terms of 
spatial extent (from local to global) or in terms of amount of information to be processed when individualizing information (from global to local).

\subsection{Terrain: Mapping Terrestrial Biodiversity}

Terrestrial biodiversity provides an interesting case considering phenomena on land. The UN Convention on Biodiversity (CBD) and the EU have set a new target of halting the loss to biodiversity by the year 2020. Achieving this goal in the first place requires a solid basis and a new approach to current judging progress. Observational data on biodiversity has to be merged from all available sources while assuring high quality. Outreach groups for data survey can greatly widen the base from which observational data may be gleaned. This data can then be merged with existing data from research organizations as well as historic data available from museums. Scenarios on reporting biodiversity illustrate how humans that are supported by mobile devices, such as smart phones, can act as the main 'sensor' for data provision.

In order to maintain data quality when integrating data from multiple sources, future eEnvironment services will have to include mechanisms for context aware quality assurance of reported data. The initial quality assurance processing should at least account for:

1. Spatial probability, i.e. does this species fit into this bio-geographical region?

2. Temporal probability, i.e. can it be observed at this time of the year?

3. Comparison with common mis-identifications, i.e. providing the user with image of other types often confused with type identified - does the leaf of this species look like this?

Such a quality assurance process requires a semantic backbone for:

- Structuring and storage of taxonomic information, including both scientific and common species names.

- Storing additional species information as required for context aware quality assurance, i.e. bio-geographical regions of occurrence, types often wrongly identified as.

- Semantic mapping between species lists, against common opinion, scientific species names are not completely standardized across Europe.

- Semantic annotation of external expert knowledge, where a wide pool of external expert, for correct identification of species sighted, can be leveraged through crowd-sourcing mechanisms.

\subsection{Atmosphere: Monitoring Individualized Pollen Exposure}

On the atmospheric sphere, we concentrate on individual exposure assessment and air quality/meteorological alerts. Today, we have easy access to a great deal of information via television, radio and the World Wide Web. This includes pollution, pollen and meteorological data which are all relatively easily accessed in one or more dissemination channels. All this data contributes to a common sense, but it is not tailored to an individual user's needs. Relevancy of data and interpreting it are key issues for users today, especially with regards to pollen and pollution which directly affect as many as $25 \%$ of the population. 
Future eEnvironment services should therefore aid individuals in tailoring information relevant to their specific requirements by providing personalized threshold alerts for air quality, meteorological conditions and pollen, as well as enabling the users to feed data back into the system which will then be used to further enhance the relevancy of the data to the user (Figure 1).

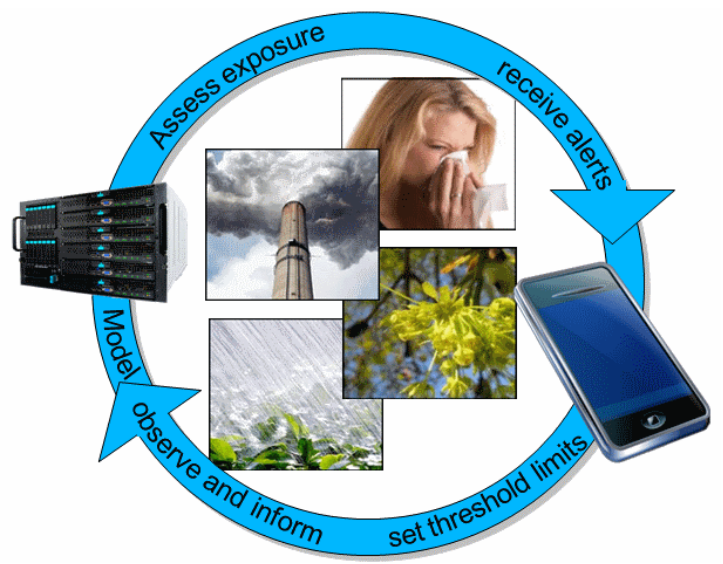

Fig. 1. Desired eEnvironment Services in the air quality domain

This personalization of the information becomes more achievable due to the increasing prevalence of GPS enabled 'smart' phones which will enable the system to assess the individual's exposure to pollution by recording the individual's coordinates and matching them with actual observation data with a higher degree of accuracy than is generally available to the public. In addition, by taking advantage of easier to use interface design we will enable the individual users to become part of the web of sensors by providing mechanisms for Voluntary Geographic Information (VGI) [9]. This data will feed back into the system and provide another layer of information to further enhance the existing infrastructure. An example of VGI could be a user reporting the prevalence of rag weed (to which he might be allergic) in an area where for which no data currently exists, thereby potentially alerting other sufferers of the existence of the allergenic plant.

\subsection{Marine: Managing Marine Resources}

The EU's marine waters support a wide range of economic and social activities. The Integrated Maritime Policy for Europe seeks to realize the significant potential of Europe's vast marine resources through the alignment of research and innovation capacity with relevant policy and market requirements. The Marine Strategy Framework Directive (MFSD) establishes a legally binding framework within which Member States shall take the necessary measures to achieve or maintain good environmental status in the marine environment by the 2020. The Directive constitutes the vital environmental component of the Union's future maritime policy, and is designed to achieve the full economic potential of oceans and seas in harmony with the marine 
sphere. The scale of the challenge is reflected in range of current monitoring requirements for the European marine environment which includes 70,000 km of coastline and an associated investment required of over $€ 1$ billion a year [10].

As new standards and approaches to the collection and management of marine data emerge $[11,12,13]$ the next generation of decision based management tools must scale to transcend national borders and facilitate an ecosystem approach to the development of Europe's marine resources and related sectors including offshore energy, environmental monitoring, aquaculture and marine tourism.

Current observations of the marine environment are achieved using a variety of commercially available sensors deployed on static and mobile platforms both above and below water. Measurements can be taken from in-situ, air-borne, water-borne, space-borne or even human-borne sensing methods to measure various meteorological, oceanographic, geophysical and biological processes. The measurement spectroscopy used in these observations can also vary (UV, IR, visible, digital, analog etc.). Mission critical data includes information on meteorological and oceanographic data (e.g. wind, sea level pressure), sea states (namely wave conditions and water movements) and information on changes in the chemical status of the water. As a consequence, a generic approach is required for the description and integration of heterogeneous sensors and sensor data regarding marine observations. Challenges for the integration and interpretation of harvested data also include the ability to deliver large volumes of real time streaming data from advanced sensing platforms such as acoustic (e.g. hydrophones, sonar, ADCP, etc.) and video feeds. The ability to dynamically scale the response of distributed sensor networks in response to unpredictable environmental events is also likely to act as a key enabler in the realization of real time dynamic marine monitoring networks and the development of a range of associated products and services for marine sector activities.

New eEnvironment services will have to consider the need for secure, smart and fast mobile communications, agent-based middleware used to empower the marine stakeholder community to observe and operate in the marine environment. Diverse and heterogeneous data and information sources should be fused and prototyped for delivery on demand using multiple distributed services and might be presented via social networking internet technologies together with sensor web enablement and fusion services technologies (Figure 2).

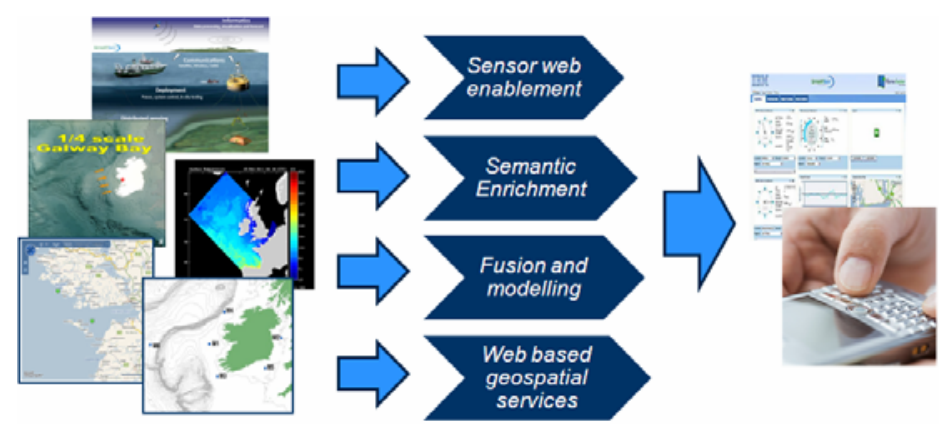

Fig. 2. Requirements for eEnvironment services for the marine sector 


\subsection{Beyond Foreseen eEnvironment Services}

Beyond the cases outlined above (section 2), which are likely to become reality in the near future, we may expect even more sophisticated applications, such as:

- A fully scalable, real-time environmental and social footprint, which provides near real-time information about individuals or groups of individuals in terms of their waste production, $\mathrm{CO} 2$ emission, water consumption, income, connections to other people and organizations etc.

- Event tracing trough observation networks, tweets and newspaper articles, in which for example natural disasters (but also political crises) are monitored from the causes, over the happening and direct responses, all the way to long term impacts, using the manifold information channels that are available.

- Social networking with sensors and environmental models, which basically enables the discovery of sensor networks, which may potentially be used as inputs to environmental models, but also serve as a communication platform for improving scientific models, and for informing about events, such as a predicted flood. This network might be even augmented with the social networking platforms of today.

Given the rapid development of ICT for environment during the last years and the growing citizen interests and technological capabilities, it is likely that also such or similar applications will become reality within this decade. We live in an era of growing user expectations on the one side and arising technological potential on the other side. Our information systems have to account for these frequent changes.

\section{Generalization and Requirements Analysis}

Given the information above and considering similar cases from other domains, we become able to extract generic components for environmental ICT applications and eEnvironment services. In this section, we introduce the roles, which are involved in generating knowledge about our environment and define the overall added-value chain. In a second step, we present common requirements for future eEnvironment services. In doing so, we provide a bridge between practical environmental applications and the wider political framework. The presented findings could equally be applied to other geospatial domains, not only to environment.

\subsection{The Value Chain of Environmental Knowledge Generation}

Analyzing the descriptions of section 2, we can extract a total of six roles, which contribute to the generation of environmental knowledge:

1. Observer, being the initial source of information about the environment. This may reach from sensor to citizen.

2. Publisher, making a resource, such as an observation, discoverable to a wider audience, e.g. by providing required resource descriptions (metadata).

3. Discoverer, being the entity that finds a resource based on all available descriptions. 
4. Service Provider, making information or an environmental model accessible to (and usable by) the wider audience.

5. Service Orchestrator, being responsible for combining existing services in a way that they create information for a distinct purpose, i.e. environmental application focusing on a particular sphere on topic.

6. Decision Maker, consuming an environmental application in order to retrieve decision supporting material and making a final decision based on the information available.

Consequently, the process workflow can be summarized as in the figure below (Figure 3). We call this workflow the added-value chain of environmental knowledge generation. Notably, following this workflow services may themselves get published in order to serve as building blocks for more complex eEnvironment solutions.

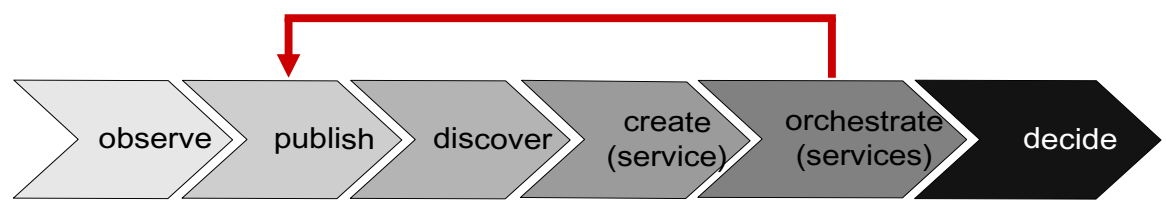

Fig. 3. The added-value chain of environmental knowledge generation

\subsection{Overview of Stakeholders}

The roles identified above (section 3.1) are played by a variety of individuals and organizations. Most of these have been mentioned in the descriptions of desired services in section 2 . In a nutshell, those can be defined as:

- Citizens of a particular social, political, or national community;

- Environmental agencies on sub-national, national and European level;

- Public authorities of national and regional and other level;

- Industries from the primary, secondary and service sector;

- Platform providers offering frameworks on which applications may be run;

- Infrastructure providers offering physical components and essential services;

- Sensor network owners holding the sensor and basic communication hardware.

Table 1 provides an overview of the manifold mappings between these stakeholders and the different roles in the value chain of environmental knowledge generation. To highlight only a few aspects: citizens can play all roles, they may even discover available information and provide new services ('mash-ups'). The decisions they may take are on individual level, such as should "I travel through an area with bad air quality?" All decisions that can be taken are strongly dependent on the stakeholder; industries may use the environmental information for logistic or location planning, while the owners of sensor networks may decide about the maintenance of their facilities. 
Table 1. The added-value chain of environmental knowledge generation

\begin{tabular}{l|cccccc} 
& observe & provide & discover & create & orchestrate & decide \\
\hline Citizens & $\mathrm{x}$ & $\mathrm{x}$ & $\mathrm{x}$ & $\mathrm{x}$ & $\mathrm{x}$ & $\mathrm{x}$ \\
Environmental agencies & $\mathrm{x}$ & $\mathrm{x}$ & & $\mathrm{x}$ & & $\mathrm{x}$ \\
Public authorities & & $\mathrm{x}$ & & $\mathrm{x}$ & & $\mathrm{x}$ \\
Industries & & & $\mathrm{x}$ & $\mathrm{x}$ & $\mathrm{x}$ & $\mathrm{x}$ \\
Platform providers & & & & $\mathrm{x}$ & & \\
Infrastructure providers & & & & $\mathrm{x}$ & & \\
Sensor network owners & $\mathrm{x}$ & $\mathrm{(x)}$ & & (x) & $\mathrm{x}$
\end{tabular}

\subsection{Requirements for a Next Generation of eEnvironment Services}

Given all this, (i) what would be the needs for a next generation of eEnvironment services in Europe and (ii) what might be a suitable framework for establishing requested platforms and infrastructures? We address the first question in the remainder of this section. We outline the cases sketched above and derive functional and nonfunctional requirements. Available frameworks for future developments are addressed in the next section (section 4).

Re-visiting the sections above, we identify at least the following needs:

- discovery, access, visualization and publication of data sets;

- discovery, access, visualization, and planning of sensor observations;

- access to environmental models and simulations as services;

- transformation of data sets and harmonization of observations;

- composition and invocation of workflows;

- support and enforcement of data and service policies based on identity, licenses, trust chains, etc.;

- provision of objective, semi-objective and subjective observations by end users;

- access and use of controlled vocabularies, taxonomies, ontologies and annotations;

- integration with the Semantic Web and Web 2.0; and

- interoperability with existing and planned infrastructures in the context of:

o the most relevant initiatives at international level, such as INSPIRE, GMES, GEOSS; and

o relevant well-established communities, including research and egovernment infrastructures.

Specific components (environmental enablers) should support these requirements. They should be designed and developed leveraging existing architectural approaches and technical specifications, and re-using/extending existing tools. Particular attention should be paid to open international standards and communities-of-practice specifications, and to open source components in order to make the resulting system more flexible and scalable (see also [14]). 


\section{Future Internet: A Framework for Implementing Change}

In response to the world economic crisis in 2008, the European Commission formulated its long term vision in Europe 2020 strategy [3], which emphasizes actions around three main priorities: (i) developing an economy based on knowledge and innovation (smart growth): (ii) promoting a more resource efficient, greener and more competitive economy (sustainable growth); and (iii) fostering a high-employment economy delivering social and territorial cohesion (inclusive growth). Seven flagship initiatives give substance to the strategy. They address innovation, youth and the labor market, digital agenda, resource efficiency, industrial policy in the global context, skills and jobs, and social and territorial cohesion respectively. In particular, the Innovation Union [5] initiative aims to improve conditions and access to funding for research and innovation in Europe, to ensure that innovative ideas can be turned into commercial products and services that create jobs and economic growth, whereas the Digital Agenda for Europe [4] outlines policies and actions to maximize the benefit of the Digital Revolution for all. The Digital Agenda foresees the action to "work with the Member States and stakeholders to implement cross-border eEnvironment services, notably advanced sensor networks". Then again, the concept of the Future Internet [7] is part of the Digital Agenda for Europe's efforts to deliver economic benefits from fast to ultrafast Internet and interoperable applications.

Considering the latter, the European Commission (EC) provided €90 million for funding Future Internet-related research in 2011, and a further $€ 210$ million in 2012 2013 through the Future Internet Public Private Partnership (FI-PPP) FP7 Programme. The FI-PPP aims to (i) to support an Internet-enabled service economy, (ii) to improve key ICT infrastructures of Europe's economy and society by making them better able to process massive amounts of data originating from multiple sources; (iii) to render the Internet more reliable and secure; and (iv) to allow real time information to be processed into real time services.

There is a clear opportunity to further develop the sustainability-innovation-growth triangle by dedicating a usage area of the FI-PPP to the environment. This provides an excellent frame for addressing the required environmental enablers, which have been identified in section 3. For optimizing the outcomes, the early and strong involvement of application areas from all environmental spheres is essential. Only in this manner, the current push of technological approaches can be balanced with applications' need, which eventually will lead to powerful and useful eEnvironment solutions.

\section{Summary and Outlook}

This paper outlined some changes, which are required for improving eEnvironment services. Three concrete cases were presented covering the terrestrial, marine and atmosphere domain; those were complemented by a set of more futuristic applications. Analyzing the various descriptions, we extracted a total of six involved roles and depicted a general workflow for environmental knowledge generation. Stakeholders have been identified for each of these roles and a set of requirement environmental enablers has been presented. The FI-PPP has been identified as a suitable frame for implementing the requested changes in Europe. The 'application pull' of requirements will be key to success. If we fail to clearly formulate and communicate 
these requirements we will miss a unique opportunity. We are just starting to investigate these issues and will work to analyze the underlying principles and consequences for the stakeholder communities. We hope to successfully contribute to the future of eEnvironment in this manner.

Acknowledgements. We thank the ENVIROFI (FP7 - 284898) project consortium for the lively discussions we had. This paper is based on our common findings.

\section{References}

1. Scientific Grand Challenges identified to address global sustainability - ISCU prepublication, http: / /www . icsu-visioning .org/wp-content/uploads / GrandChallenges_Pre-publication.pdf (last accessed February 12, 2011)

2. Craglia, M., Goodchild, M., Annoni, A., Camara, G., Gould, M., Kuhn, W., Mark, D., Masser, I., Maguire, D., Liang, S., Parsons, E.: Next-generation digital earth: A position paper from the vespucci initiative for the advancement of geographic information science. International Journal of Spatial Data Infrastructures Research 3, 146-167 (2008)

3. Official Homepage of the European Commission Europe 2020 Strategy, http : / / ec. europa.eu/europe2 020 /index_en.htm (last accessed March 25, 2011)

4. Official Homepage of the European Commission Digital Agenda for Europe (DG Information Society and Media), http: / /ec.europa.eu/information_society/ digital-agenda/index_en.htm (last accessed March 25, 2011)

5. Official Homepage of the European Commission Innovation Union, http: / / ec.europa.eu/research/innovation-union/index_en.cfm (last accessed March 25, 2011)

6. Accordino, F.: Digital Science and its impact on Scientific Societies. Presentation given at European Computer Science Summit Prague (CZ) (October 13)

7. European Future Internet Portal, http://www. future-internet.eu/ (last accessed March 25, 2011)

8. Hřebíček, J.(Chief ed.), Hradec, J., Pelikán, E., Mírovský, O., Pillmann, W., Holoubek, I., Bandholtz, T. (eds.) Proceedings of the European conference of the Czech Presidency of the Council of the EU TOWARDS eENVIRONMENT. Opportunities of SEIS and SISE: Integrating Environmental Knowledge in Europe. Masaryk University, Brno, Czech Republic (March 2009)

9. Goodchild, M.: Citizens as sensors: the world of volunteered geography. GeoJournal 69(4), 211-221 (2007)

10. Marine Knowledge 2020, MEMO/10/404, http: / / europa . eu/rapid/ pressReleasesAction . do? reference=MEMO / $10 / 404 \&$ format $=$ HTML \&aged $=0 \&$ language $=E N \& g u$ i Language $=e n($ last accessed March 25, 2011)

11. Official Homepage of the Pan-European infrastructure for Ocean \& Marine Data Management (SeaDataNet) project, http://www. seadatanet.org/ (last accessed March 25, 2011)

12. Official Homepage of the Water Information System for Europe (WISE) project, http: / /water. europa. eu / (last accessed March 25, 2011)

13. Official Homepage of the European Marine Observation and Data Network (EMODNET), http: / / ec.europa.eu/maritimeaffairs/emodnet_en.html (last accessed March 25, 2011)

14. Interoperable Solutions for European Public Administrations (isa): European Interoperability Strategy (EIS) for European public services (December 16, 2010) 